\title{
Poor Metabolic Quality Embryo is Capable to Implant - A Case Report
}

\section{Bartmann $\mathrm{AK}^{* 1,2,3}$, Silva LFI ${ }^{1,4}$, Silva LLM ${ }^{2}$, Mello DFRE ${ }^{2}$, Gualberto JR ${ }^{2}$, Bertoncelo $\mathrm{DM}^{2}$, Sanchez $\mathrm{IG}^{2}$,} Millani KVS ${ }^{2}$ and Contini SHT ${ }^{3}$

\author{
${ }^{1}$ Human Reproduction Center of the Ana Bartmann Clinic, Brazil \\ ${ }^{2}$ Medical School, University of Ribeirão Preto (UNAERP), Brazil \\ ${ }^{3}$ Biotechnology Department,University of Ribeirão Preto (UNAERP), Brazil \\ ${ }^{4}$ Department of Obstetrics and Gynecology, School of Medicine of Ribeirão Preto, University of São Paulo (USP), \\ Brazil
}

${ }^{*}$ Corresponding author: Bartmann AK, Electro Bonini Hospital - University of Ribeirão Preto (UNAERP), Av Leao XI, s / n CEP: 14096900, Ribeirao Preto SP, Brazil, Tel: 00551639415121, E-mail: anabartmann@uol. com.br

\section{Citation: Bartmann AK, Silva LFI, Silva LLM, Mello DFRE, Gualberto JR, et al. (2017) Poor Metabolic} Quality Embryo is Capable to Implant - A Case Report. J Gynecol Res 3(2): 201. doi: 10.15744/2454-

3284.3.201

Received Date: November 14, 2016 Accepted Date: December 27, 2017 Published Date: December 29, 2017

\begin{abstract}
Introduction: There has been little advance in embryo selection criteria since the first IVF. To this day, we select embryos by morphological criteria, which provide little information as to the actual embryo quality. The objective of this paper is to report the case of a patient who had her embryos' culture media analyzed in order to determine the pyruvate and lactate concentration, in addition to morphologic criteria.
\end{abstract}

Methodology: 33-year-old patient who underwent IVF and had six frozen embryos. We analyzed each embryo's culture medium microdroplets by Gas Chromatography coupled with Mass Spectrometer (GC/MS) in order to quantify pyruvate and lactate concentrations.

Results: Of the six embryos, one was a grade $8 \mathrm{~A}$ embryo with pyruvate uptake of $10.43 \%$ and lactate production of $2.5 \%$ and one was a grade $8 \mathrm{~B}$ with pyruvate uptake of $10.54 \%$ and lactate production of $3.11 \%$ - both did not survive thawing. Two grade $8 \mathrm{~B}$ embryos with pyruvate uptake of $9.91 \%-8.81 \%$ and lactate production of $0.84 \%-0,92 \%$ were transferred without implantation. The remaining embryos were then transferred - a $6 \mathrm{~B}$ embryo with pyruvate uptake of $8.41 \%$ and lactate production of $0.0 \%$ and a $7 \mathrm{~B}$ embryo with pyruvate uptake of $8.75 \%$ and lactate production of $0.39 \%$. One resulted in gestation.

Conclusions: This case showed that embryos with low pyruvate uptake and lactate production may have good implantation potential.

Keywords: Metabolites; Culture medium; IVF; GC-MS

\section{Introduction}

Assisted Reproduction (AR) is an area of knowledge that has constantly developed over the years. Nevertheless, the success rate of AR treatments is of about $35 \%$ to $40 \%$ [1] and little improvement has been made regarding embryo selection criteria. We still use purely morphological criteria to select embryos, such as symmetry and number of blastomers in each embryo. Such classification tells us little about these embryo's true implantation potential $[2,3]$.

There are several studies underway looking for new ways of embryo selection. As a strong predictor of embryo quality, we point out their metabolic activity, seeing that embryo viability and implantation potential are closely related to the embryos' metabolism [2]. Since the morphological score gives us little information on embryo quality, it is common to see embryos with excellent scores that do not implant and embryos with bad scores that result in beautiful babies. Thus, the current trend is to associate morphologic and metabolic criteria to select embryos.

We know that the embryo produces energy through aerobic and anaerobic metabolic pathways [4]. For that reason, we highlight the role of pyruvate and lactate on the assessment of embryo quality.

Experiments carried out in the decades of 1950 and 1960 showed that the embryos of mice were unable to use glucose as an energy source before their 8-cell stage [4]. Biggers and colleagues demonstrated in 1967 [5], that there is a glycolytic pathway restriction during the development of oocytes. As such, the energy produced after fertilization and during the initial development of the embryo is restricted to the use of pyruvate, a by-product of glycolysis that is directly converted into Acetyl Coenzyme A and used 
in the Krebs cycle [6]. Pyruvate is mainly consumed by the embryo at the morula stage [7,8]. Moreover, pyruvate is the only source of energy used by the embryo in its first mitotic division and for this reason it is essential for its development during the period that immediately follows fertilization [5].

Increased amounts of lactate are synthesized in embryo development [9]. Research suggests that high lactate production by embryos in their pre-implantation stage is an adaptation to culture conditions and the higher the production the better the adaptive conditions.

Gardner et al. 2001 [10] - suggest that the differences in the use of pyruvate or lactate at different stages of development are due to differences in the regulation of lactate dehydrogenase (LDH). This enzyme catalyzes the lactate oxidation by a NAD+ molecule, leading to the formation of pyruvate and NADH and vice versa, allowing the cell to obtain pyruvate from a single reaction, simpler than the series of reactions required for glycolysis.

This being the case, we conclude that the greater the metabolic capacity of an embryo the better its quality. Accordingly, when we analyze its biochemistry, an embryo with high metabolic capacity must a priori present a low concentration of pyruvate and a high concentration of lactate.

The objective of this paper is to report the case of a low metabolic embryo implantation.

\section{Case presentation}

\section{Patient's Identification}

In this article, we report a case of a 33-year-old patient, married to a 29-year-old man, who underwent controlled ovarian hyperstimulation (COH) with standard protocol and ICSI [11]. Recombinant FSH therapy (Gonal-F, Serono Pharmaceuticals, Boulogne, France) was initiated at a dosage of $225 \mathrm{IU} /$ day for 10 days, until the day of hCG administration (Ovidrel, Organon Pharmaceuticals, Saint-Denis, France). Administration of hCG was performed as soon as $\geq 4$ pre-ovulatory follicles (16-22 mm in diameter) were observed. Oocyte retrieval was performed 34 hours after trigger. We collected 6 oocytes from 7 follicles. All oocytes fertilized and became morphologically normal embryos.

Since she did not have endometrium thickness $>8 \mathrm{~mm}$, we decided to freeze all embryos for further transfer. Patient had no endometrial phatology or systemic disease. The cause of infertility was male factor (moderate oligospermia).

\section{Materials and Methods}

At the end of $\mathrm{COH}$, six oocytes were collected and the ICSI procedure was performed, resulting in six embryos. The embryos were cultured in individual microdroplets of a Global Life Total ${ }^{\circledR}$ (Life Global ${ }^{\oplus}$, Guilford-CT, USA) medium for three days without washing. On the first day of culture (D1), a falcon 353002 dish was prepared with six side microdroplets and a central microdroplet with $40 \mathrm{uL}$ of Life Global Total ${ }^{\circledR}$ supplemented medium, covered with $5 \mathrm{~mL}$ of mineral oil (Irvine Scientific - Santa Ana, CA). The embryos were cultured within the six microdroplets. The central microdroplet remained without embryo culture for three days (control droplet). In the morning of the third day (D3), the embryos were photographed and morphologically assessed according to Veeck's criteria [12]. They were placed in a new culture dish. The microdroplets of the previously cultured dish were separately collected after complete removal of mineral oil. We analyzed them by evaluating their pyruvate and lactate levels, first in the central droplet (without embryo culture) and subsequently in the other microdroplets where culture took place. We compared the metabolic levels of the central microdroplet and of the microdroplets that had had culture, and evaluated the consumption and production of the metabolites found, establishing a metabolic profile of each embryo. As the patient had thin endometrium, all embryos were thawed for further intra-uterine transfer.

For thawing, we used a thaw kit (Irvine Scientific - Santa Ana, CA) containing: (i) equilibration solution (ES): $7.5 \%$ (v/v) ethylene glycol $+7.5 \%(\mathrm{v} / \mathrm{v})$ dimethylsulphoxide (DMSO) in an M-199 HEPES-buffered medium supplemented with $20 \%$ dextran serum supplement (DSS); and (ii) vitrification solution: $15 \%$ (v/v) ethylene glycol + 15\% (v/v) DMSO + 0.5 mol/l sucrose [13].

The clinical monitoring of lactate and pyruvate by gas chromatography technique coupled with mass spectrometry (GC/MS) required a prior derivatization of the sample; therefore we added $1 \mathrm{~mL}$ of ethanol to $30 \mu \mathrm{L}$ of medium followed by centrifugation. The supernatant was transferred to a glass vial and evaporated. The material was redissolved in $50 \mu \mathrm{L}$ of pyridine, $50 \mu \mathrm{L}$ of the silanizing reagent BSTFA (bis(trimethylsilyl)trifluoroacetamide - SUPELCO), 20.5 $\mu$ l of TMCS (98\% (Chloromethyl)trimethylsilane - ALDRICH), and heated to $50{ }^{\circ} \mathrm{C}$ for 15 minutes [14]. After silanization, we dosed these metabolites concentrations in the culture medium by GC/MS technique [15].

In the first transfer after endometrial preparation, two embryos did not survive thawing and two others were transferred, but did not result in gestation.

For warming, we used the same thaw kit (Irvine Scientific - Santa Ana, CA). The kit comprised: (i) a thawing solution (TS) containing $1 \mathrm{~mol} / 1$ sucrose in an M-199 HEPES-buffered medium supplemented with 20\% DSS; (ii) a dilution solution (DS) containing $0.5 \mathrm{~mol} / \mathrm{l}$ sucrose in an M-199 HEPES-buffered medium supplemented with 20\% DSS; and (iii) a washing solution (WS) containing M-199 HEPES-buffered medium supplemented with 20\% DSS [13].

In the first transfer after endometrial preparation, two embryos did not survive thawing and two others were transferred, but did 
not result in gestation. In the second transfer after a new endometrial preparation, the two remaining embryos were transferred, resulting in a single gestation. The transfers were performed after standard endometrial preparation with the use of estrogen and subsequent maintenance of the endometrium with progesterone. The two transfers were performed by the same physician, with endometrial thickness greater than $8 \mathrm{~mm}$.

The research was carried out at the Human Reproduction Center of the Ana Bartmann Clinic in partnership with the University of Ribeirão Preto (UNAERP), between August 2014 and June 2015. This case is part of a group of patients from a prospective controlled research that is currently underway. The ethical approval protocol was approved by the medical ethic committee of the hospital. Each couple whose embryos were enrolled into the study signed a written informed consent.

\section{Results}

Of the six embryos obtained by ICSI procedure (Table 1 and Figure 1), there was: a grade 8A embryo with pyruvate uptake of $10.43 \%$ and lactate production of $2.5 \%$ (compared against the control droplet) that did not survive heating. A grade $8 \mathrm{~B}$ embryo with pyruvate uptake of $10.54 \%$ and lactate production of $3.11 \%$ (against the control droplet), that also did not survive heating. A grade $8 \mathrm{~B}$ embryo with pyruvate uptake of $9.91 \%$ and lactate production of $0.84 \%$ (against the control droplet). A grade $8 \mathrm{~B}$ embryo with pyruvate uptake of $8.81 \%$ and lactate production of $0.92 \%$, (against the control). These last two were transferred, but did not result in clinical pregnancy. A grade $6 \mathrm{~B}$ embryo with pyruvate uptake of $8.41 \%$ and lactate production of $0.00 \%$ (against the control droplet). A grade 7B embryo with pyruvate uptake of $8.75 \%$ and lactate production of $0.39 \%$ (against the control droplet). These two last embryos were transferred and resulted in a single-fetus pregnancy.

\begin{tabular}{|c|c|c|c|c|}
\hline Embryo & $\begin{array}{c}\text { Morphological } \\
\text { Classification }\end{array}$ & $\begin{array}{c}\text { Pyruvate } \\
\text { Concentration }\end{array}$ & $\begin{array}{c}\text { Lactate } \\
\text { Concentration }\end{array}$ & Results \\
\hline 1 & $8 \mathrm{~A}$ & $\downarrow 10.43 \%$ & $\uparrow 2.5 \%$ & did not survive thawing \\
\hline 2 & $8 \mathrm{~B}$ & $\downarrow 10.54 \%$ & $\uparrow 3.11 \%$ & did not survive thawing \\
\hline 3 & $8 \mathrm{~B}$ & $\downarrow 9.91 \%$ & $\uparrow 0.84 \%$ & implantation failure \\
\hline 4 & $8 \mathrm{~B}$ & $\downarrow 8.81 \%$ & $\uparrow 0.92 \%$ & implantation failure \\
\hline 5 & $6 \mathrm{~B}$ & $\downarrow 8.41 \%$ & $0 \%$ & likely to be implanted \\
\hline 6 & $7 \mathrm{~B}$ & $\downarrow 8.75 \%$ & $\uparrow 0.39 \%$ & likely to be implanted \\
\hline
\end{tabular}

Table 1: Morphologic and metabolic analysis of the collected embryos. Pyruvate and Lactate concentrations are increased or decreased in relation to the control droplet (no embryo)
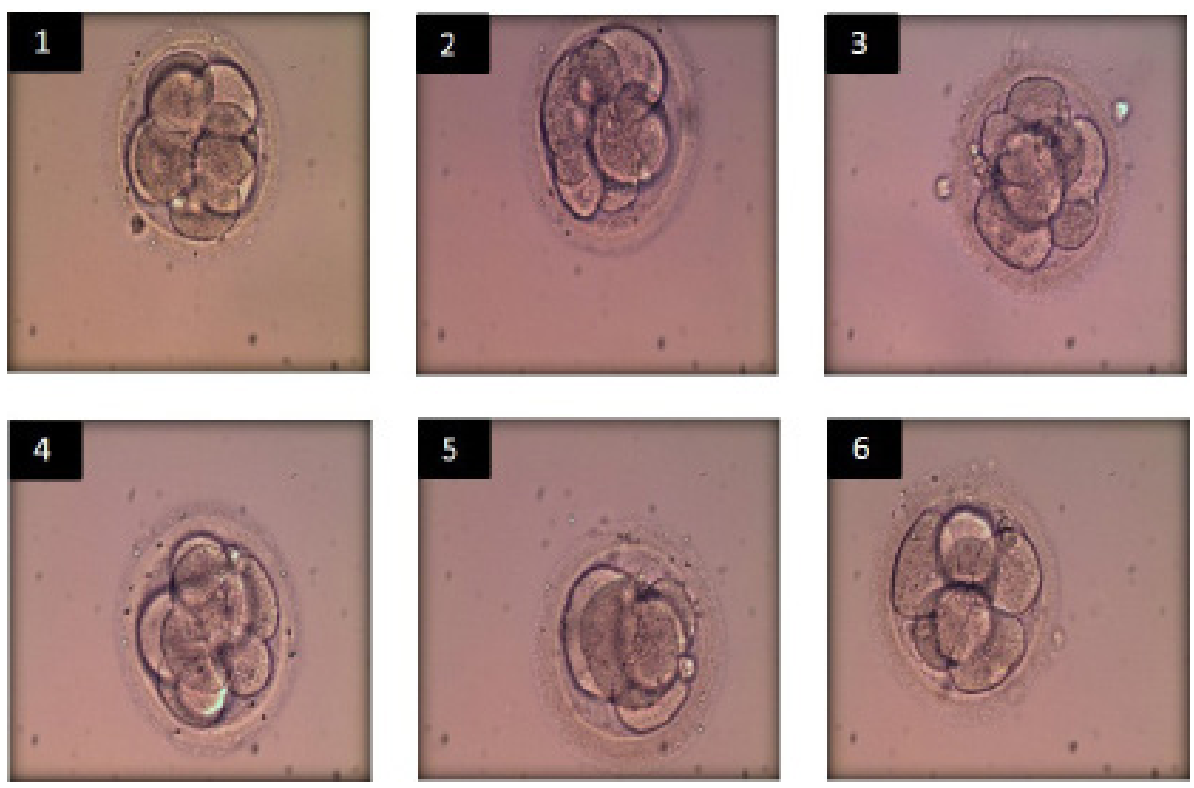

Figure 1: Morphology of the collected embryos (day 3) before thawing

\section{Discussion}

Although it is not possible to draw conclusions based on a single case report, we may notice that embryos with high pyruvate uptake and lactate production showed an inverse relationship between implantation potential and clinical pregnancy. Additional studies are required to validate these observations, but some considerations must be made. The first is related to the concept of "best embryo". Studies with a "time lapse" methodology, for example, have shown that embryos with high developmental speed have greater implantation potential [16]. It is not known, however, if high metabolic profile is related to greater implantation rates or greater cellular distress, which can influence the development of embryos [17]. A low metabolic rate, therefore, could be a positive characteristic in terms of response to the stress of cryopreservation [18]. 
Besides the importance of evaluating the metabolic rate of the embryo, it is known that the study of its genetic is an inherent condition for implantation success [19] Corroborating these data, the epigenetic plays an important role in embryo cellular signaling as well as in embryo programming in response to environmental disturbances [20].

It is important to recognize that there are many variables, which can play a role in human embryo implantation and puzzle the interpretation of data. This means that it is soon to state that a good embryo has high metabolic rate. More studies are needed to establish what a good embryo mean in terms of metabolism. It is possible that the rates of pyruvate uptake and lactate production do not need to be as high as theory suggests. Or that the proportions between metabolites are more important than their isolated concentrations.

\section{Conclusion}

We conclude that the metabolic rate analyzed alone is not a good predictor of implantation, since, as we showed, embryos with poor metabolic rate are also capable to implant.

The excess of nutrients as well as their lack may be harmful to the development of embryos. Thus, it is of the utmost importance to understand the metabolic basis of pre-implantational stage in order to reduce the stress to which embryos are submitted during IVF procedures.

\section{Consent}

A written informed consent was obtained from the patient for the publication of this case report and any accompanying images. A copy of the written consent is available for review by the Editor-in-Chief of this journal.

\section{Competing interests}

The authors declare no competing financial interests. The study was conducted in the Gynecology and Obstetrics Department of the University of Ribeirão Preto (UNAERP) in partnership with the Ana Bartmann clinic - Human Reproduction Center.

\section{Acknowledgements}

We thank the professionals of the Electro Bonini Hospital and the Human Reproduction Center of the Ana Bartmann Clinic for their cooperation.

\section{Reference}

1. Kissim DM, Kulkarni AD, Kushnir VA, Jamieson DJ (2014) Number of Embryos Transferred after in vitro fertilization and good perinatal outcome. Obstet Gynecol 123: 239-47.

2. Dzik, Pereira DHM, Amaral WN (2012) Tratado de Reprodução Assistida. 2: 317-26.

3. Devreker F (2007) Uptake and release of metabolites in human preimplantation embryos Human Preimplantation Embryo Selection. In: Cohen J, Elder K. Human Preimplantation Embyo Selection. London: Academic 325-36.

4. Whitten WK (1957) Culture of tubal ova. Nature 179: 1081-2.

5. Biggers JD, Whittingham DG, Donahue RP (1967) The pattern of energy metabolism in the mouse oocyte and zygote. Proc Natl Acad Sci 58: 560-7.

6. Botros L, Sakkas D, Seli E (2008) Metabolomics and its application for non-invasive embryo assessment in IVF. Mol Human Reprodu 14:679-90.

7. Turner K, Martin KL, Woodward BJ, Lenton EA, Leese HJ (1994) Comparison of pyruvate uptake by embryos derived from conception and non-conception natural cycles. Human Reprod 12: 2362-6.

8. Conaghan J, Hardy K, Handyside AH, Winston RM, Leese HJ (1993) Selection would criteria will be human embryo to transfer: comparison of pyruvate uptake and morphology. J Assist Reprod Genet 10: 21-30.

9. Gott AL, Hardy k, Winston RML, Lees HJ (1990) Non-invasive meansure of pyruvate and glucose uptake and lactate production by single human preimplantation embryos. Hum Reprod 5: 104-8.

10. Gardner DK, Lane M, Stevens J, Schoolcraft WB (2001) Noninvasive assessment of human embryo nutrient consumption as a measure of developmental potential. Fertil Steril 76: 1175-80.

11. Jungheim ES, Meyer M, Broughton DE (2015) Best practices for controlled ovarian stimulation in IVF. Semin Reprodu Med 33: 77-82.

12. Rosenwaks Z, Veeck LL, Liu HC (1986) Pregnancy following transfer of in vitro fertilized donated oocytes. Fertil Steril 45: 417-20.

13. Stoop D1, De Munck N, Jansen E, Platteau P, Van den Abbeel E, et al. (2012) Clinical validation of a closed vitrification system in na oocyte-donaytion programme. Reprod BioMed Online 24: 180-5.

14. Villas-Bôas SG, Delicado DG, Akesson M, Nielsen J (2003) Simultaneous analysis of amino and nonamino organic acids as methyl chloroformate derivatives using gas chromatography-mass spectrometry. Anal Biochem 322: 134-8.

15. Glamočlija J, Soković M, Tešević V, Linde GA, Colauto NB (2011) Chemical characterization of Lippia alba essential oil: an alternative to control green molds. Braz J Microbiol 42: 1537-46

16. Bulgarelli DL, Ting AY, Zelinski MB (2013) Vitrification of secondary isolated follicle: a new option for fertility preservation in cancer patients. JBRA Assisted Reproduction doi: 10.5935/1518-0557.20130068.

17. Geber S, Elielton RC, Marcello V, Marcos S (2006) Metabolismo Embrionário no Período pré-implantação. Centro de Medicina Reprodutiva 34: 551-7.

18. Tatone C, Di Emidio G, Vento M, Ciriminna R, Artini PG (2010) Cryopreservation and oxidative stress in reproductive cells. Gynecol Endocrinol 26: 563.-7.

19. Nascimento VCPS, Melo AS, Berteli TS, Bartmann AK (2014) The Importance of the Study of the Embryonic Metabolome in Assisted Human Reproduction. JBRA Assist Reprod 18: 151-4. 
20. Leese HJ (2015) History of oocyte and embryo metabolism. Reprod Fertil Dev 27: 567-71.

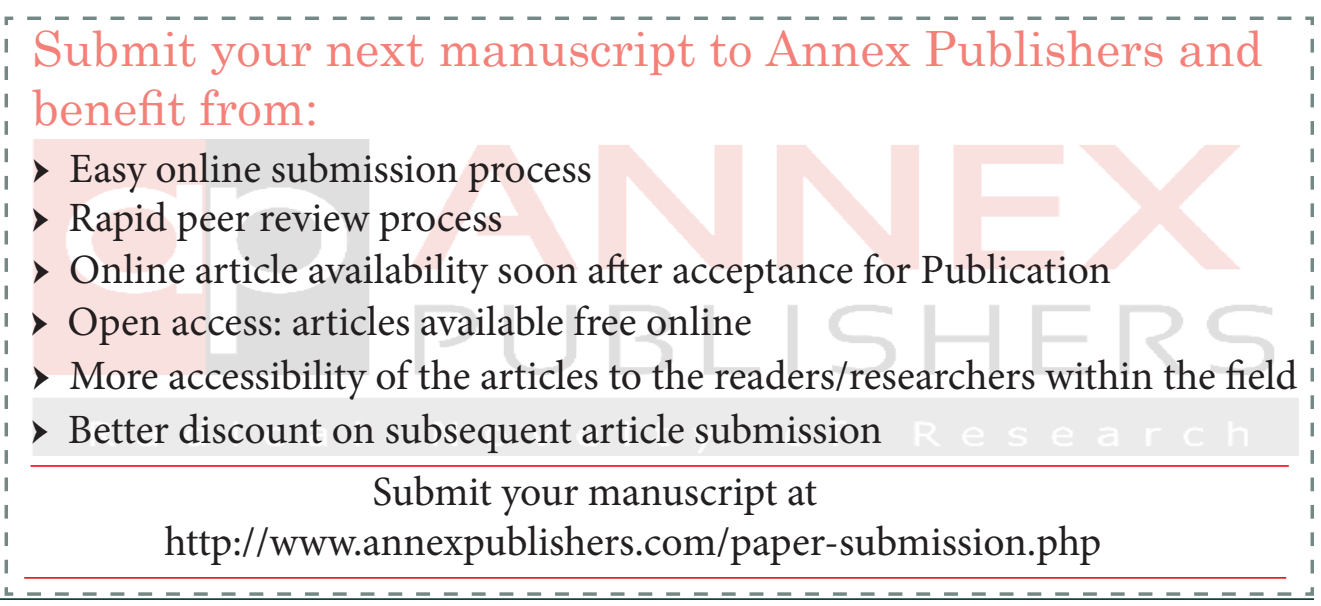

